\title{
Phenomenology of COPD: interpreting phenotypes with the ECLIPSE study
}

\author{
Alberto Papi1, Maria S. Magnoni², Carmelo C. Muzzio², Gianmarco Benso², Andrea Rizzi² \\ 1 University of Ferrara, Department of Respiratory Diseases Unit, Department of Medical Sciences, Ferrara \\ 2 Medical and Scientific Department, GlaxoSmithKline, Verona, Italy
}

\begin{abstract}
The Evaluation of COPD Longitudinally to Identify Predictive Surrogate End-points (ECLIPSE) study was a large 3-year observational multicentre international study aimed at defining COPD phenotypes and identifying biomarkers and/or genetic parameters that help to predict disease progression.

The study has contributed to a better understanding of COPD heterogeneity, with the characterization of clinically important subtypes/phenotypes of patients, such as the frequent exacerbators or patient with persistent systemic inflammation, who may have different prognosis or treatment requirements.

Because of the big amount of information that is starting to be produced from metabolomic, proteomic and genomic approaches, one of the biggest challenges is the integration of data in a biological prospective such as clinical prognosis and response to medicinal products. In this article we highlight some of the progress in phenotyping the heterogeneity of the disease that have been made thanks to the analyses of this longitudinal study.
\end{abstract}

\section{Introduction}

Chronic obstructive pulmonary disease (COPD) is defined in the GOLD guidelines as a disease "characterized by persistent airflow lim-

Corresponding author: Prof. Alberto Papi, Centro Interdipartimentale per lo Studio delle Malattie Infiammatorie delle Vie Aeree e Patologie FumoCorrelate (CEMICEF), Università degli Studi di Ferrara, Via Rampari di San Rocco, 27, 44121 Ferrara, Italy. E-mail: ppa@unife.it

Key words: COPD; phenotypes; exacerbations; lung function.

Disclosures: ECLIPSE is a study funded by GSK.

Alberto Papi has received grants, personal fees, and non-financial support from Almirall, AstraZeneca, Boehringer Ingelheim, Chiesi Farmaceutici, Dompè, GlaxoSmithKline, Guidotti, Malesci, Merck Sharp \& Dohme, Menarini, Mundipharma, Novartis, Pfizer, Takeda, Zambon.

Maria S. Magnoni, Carmelo C. Muzzio, Gianmarco Benso and Andrea Rizzi are Glaxo Smith Kline employees.

Received for publication: 20 May 2016

Accepted for publication: 05 July 2016

CCopyright A. Papi et al., 2016

Tipografia PI-ME Editrice, Italy

Monaldi Archives for Chest Disease Pulmonary Series 2016; 83:721

doi: 10.4081/monaldi.2016.721

This article is distributed under the terms of the Creative Commons Attribution Noncommercial License (by-nc 4.0) which permits any noncommercial use, distribution, and reproduction in any medium, provided the original author(s) and source are credited. itation that is usually progressive and associated with an enhanced chronic inflammatory response in the airways and the lung to noxious particles or gases' [1]. The main cause is cigarette smoke, however domestic, environmental and work place pollution may contribute to its development and worsening. Inhaled hazardous particles trigger an abnormal inflammatory response which may lead to the destruction of the parenchymal tissue and may affect the normal defences and airways mechanisms with consequent fibrosis and reshaping of the airways. All these events are clinically expressed with dyspnoea, following even minor physical exercise, and cough, and they reduce progressively the quality of the life of the patients leading, in many cases, to a high degree of disability.

COPD affects around $10 \%$ of the adult population [2] and it is predicted that it will be the third cause of death and disability in the world by the year 2020 [3].

The clinical course of COPD often presents exacerbation episodes of the disease (which may occur in some patients even several times a year) and are characterized by an acute worsening of the symptoms lasting several days, sometimes weeks [1]. At times, hospitalisation may be necessary and in the most severe cases, the use of oxygen therapy and mechanic ventilation may be required. Exacerbations are events that negatively impact the natural clinical history of the disease as they are associated with a faster decline of the lung function and a reduced survival rate.

Significant heterogeneity of clinical presentation and disease progression exists within COPD: the objective of this article is to highlight the accumulated data from the ECLISE study that may help to identify and refine patient phenotypes in COPD.

\section{Heterogeneity of COPD}

COPD is characterised by a marked heterogeneity: the patients suffering from the same functional alteration (limitation of the airflow) may present different profiles of the pathology, both from an anatomypathological perspective (emphysema, chronic bronchitis, bronchiolar reshaping, fibrosis) and clinical perspective (dyspnoea, cough, bronchial hypersecretion, exacerbations). For example, the progression of the disease, in terms of functional decline, is not homogeneous but is affected by several factors such as exacerbation history, degree of bronchial obstruction and age [4].

The configuration of a heterogeneous disease can make the diagnostic and therapeutic approach very challenging. For this reason, the scientific community is showing an increased interest in characterising the clinical and physio-pathological heterogeneity in phenotypes within the COPD.

The concept of phenotype in reality is not new. In the second half of the past century, COPD was differentiated into 'phenotype A', characterised by a finding of emphysema, and 'phenotype B', characterised by chronic bronchitis, based on clinical, radiological and physio-pathological elements [5]. These two types represented the two extremes of a spectrum with the majority of patients positioned somewhere in the 
middle. Therefore, this distinction did not find a widespread application and was partially abandoned.

In the ' 80 s, Gordon et al. [6] proposed the Venn non-proportional diagram to indicate the possible causes, associated in various ways with each other, of a non-reversible obstruction of the airflow: based on this approach, a continuous spectrum of pathological conditions - chronic bronchitis, emphysema, asthma - with a different participation of the physio-pathological mechanisms, leads to multiform clinical patterns. The limitation of this type of theoretical differentiation consists mainly in the fact that there are no validated objective parameters on which to base the differential diagnostics and the prognosis.

Progress made in recent years in diagnostic imaging (high resolution computerised tomography, magnetic resonance) has contributed considerably to a renewed interest in the classification of patients based on clinical-pathological phenotypes, while providing morphological, quantitative and topographical documentation that is more precise, faster and more standardised compared with the old Gold-standard represented by pathological anatomy. These considerations have led to new studies regarding the classification of the COPD clinical phenotypes.

\section{What is a phenotype?}

A phenotype is the expression of a set of 'characters' specific to a person (morphological, behaviour, and other characteristics) and is the result of interactions between an individual's genetic heritage (genotype) and environmental factors. In the case of many diseases, such as COPD, the interaction between environmental factors (smoke, dust at the workplace) and the host's constitutional factors (affected by the genetic make-up) may determine the different clinical aspects or clinical phenotypes of the disease, e.g. susceptibility to exacerbations, speed in the progression of the disease, hyperproduction of mucus.

The clinical characteristics of a phenotype should be prospectively validated and should have a predictive value, thus providing important information for the prognosis and treatment plans. In this context, an important aspect is the search for biomarkers that can not only differentiate a subject with an active pathology from a healthy subject, but also identify the clinical sub-types (or phenotypes), predict the severity or progression of the disease as well as the response to the treatment.

The above considerations have reopened the research in the phenotyping of the disease adopting a method based on the cluster analysis of the potentially important variables and main components of the disease.

With this approach, Pistolesi et al. have reverted to the description of the two clinical phenotypes of Burrows [5] and on the basis of the multivariate analysis of 9 parameters (clinical, morphological, functional) have validated the subdivision of patients into two groups with characteristics corresponding to two COPD phenotypes, one with prevalent obstruction of the airways and the other with prevailing characteristics of destruction of the pulmonary parenchyma [7].

Other studies were based on the cluster analysis which involves the grouping and classification of data that are initially unclassified. The study of Burgel et al., carried out on a cohort of 322 patients with mild to very serious COPD, has identified 4 clinical phenotypes, not identifiable according to the GOLD stratification, based on the analysis of clinical variables such as cumulative exposure to smoke, FEV1, BMI, exacerbations, dyspnoea, health conditions, depression [8]. Patients with a similar degree of functional limitation may belong to different phenotypes and show significant differences in terms of age, symptoms, co-morbidity and risk of death. Similar considerations have emerged also from the Weatherhall et al. study which differentiated 5 groups of patients with chronic obstruction of the airways, including one patient with intermediate characteristics between chronic asthma and COPD [9].

The results reflect not only the complexity and heterogeneity of the disease but can also have implications in the clinical research: the benefit may depend not only on the efficacy of the treatment but also on the level of responsiveness of the different phenotypes. Garcia-Aymerich et al., in their recent PAC-COPD (Phenotype and Course of COPD) [10] study, followed for 4 years a cohort of 342 patients who had been hospitalised for the first time for COPD exacerbation, and collected clinical, physio-pathological, imaging and functional data, including spirometric measurements, stress tolerance, inflammation, quality of life, pulmonary CAT scan, microbiology of the sputum, cardiocirculatory stress test: the Authors identified 3 clinically relevant COPD phenotypes: i) COPD with a severe respiratory component, characterised by a high frequency of exacerbations and an increased risk of death compared with the other groups; ii) COPD with a moderate respiratory component compared with group 1 but a similar alteration of the pulmonary density; iii) COPD with a systemic component, characterised by a high prevalence of obesity, cardiovascular diseases and high values in inflammatory markers.

\section{The ECLIPSE study}

A fundamental contribution to the knowledge of COPD phenotypes derives from the ECLIPSE [11] (Evaluation of COPD Longitudinally to Identify Predictive Surrogate Endpoints) study which, contrary to the above mentioned studies, has explored the heterogeneity of the disease with a so-called 'unbiased' approach, i.e. without pre-defined hypotheses. It is an observational, longitudinal study, of a 3 year duration, carried out on a population of about 2180 patients with mild to very severe COPD (II - IV GOLD stages) and 566 control patients (smokers and non-smokers) for the purpose of defining the clinically relevant COPD sub-types and identifying parameters and biomarkers capable of predicting the progression of the disease [11].

The ECLIPSE cohort is one of the broadest and best characterised cohorts in COPD studies, since subjects underwent a wide range of tests including CT of the thorax in order to assess the severity and distribution of emphysema, spirometric and plethysmographic exams, several questionnaires, tests to measure stress tolerance, measurements of markers in the blood, urine and induced sputum. In addition, data reported by the patients concerning respiratory symptoms, medicinal products, tobacco consumption history, work-related exposure and concomitant pathologies were documented through a consolidated ATS-DLD (American Thoracic Society-Division of Lung Disease) questionnaire.

The cross-sectional analysis of baseline data has shown that the patients with COPD, compared to control subjects, show more frequently co-morbidity, especially of a cardiovascular nature [11]. The emphysema level correlates with the GOLD stratification, whereas the correlation between spirometric parameters and clinical signs (dyspnoea, stress tolerance, exacerbations) was very limited as a further confirmation of the fact that the degree of obstruction is not sufficient to capture the complexity and heterogeneity of the disease [11].

\section{The 'frequent-exacerbation' phenotype}

Since information about the incidence of exacerbations and the identification of the predictive factors in patients with a different level of severity of the disease is limited, one of the first analysis of the longitudinal data collected from the ECLIPSE cohort has verified 
the hypothesis that the frequency of the exacerbations may identify a specific COPD phenotype, independently from the severity of the disease [12]. Exacerbations have been defined as events that have led a physician to prescribe antibiotics or corticosteroids (or both) or that have led to hospitalisation (severe exacerbations).

The results confirm that with the worsening of the disease, the exacerbations become more frequent and severe, but also show that a large proportion of patients experience frequent exacerbations, more than one a year, independently from the degree of severity defined on the basis of a spirometric exam of the pulmonary function (Figure 1).

In the moderate stage of the disease (GOLD II), exacerbations occur in $22 \%$ of cases, $33 \%$ in the severe stage (GOLD III) and $47 \%$ in the very severe stage (GOLD IV). In these patients, the frequency of exacerbations has remained stable during the 3 year observation and a previous history of exacerbations proved to be the most predictive factor of their frequency.

Thanks to the ECLIPSE study, for the first time a distinct COPD phenotype with high risk of exacerbation was described, and purposely defined as a 'requent exacerbation', stable over time, cross-sectional with respect to the GOLD stages, characterised by an intrinsic susceptibility to triggers, such as viral infections, clinically predictable and identifiable through clinical history: with the worsening of the disease, the percentage of these 'frequent exacerbation' patients increases progressively; however, even in the moderate stage, they represent a relevant portion, i.e. $22 \%$ : since the moderate COPD is more prevalent than the severe one, the overall burden of the exacerbations may be greater in the case of the moderate disease.

A recent analysis on severe exacerbations of COPD requiring hospital admission (occurring across all stages of airflow limitation) indicates that they are a significant prognostic factor of reduced survival. COPD patients at a high risk of hospitalization can be identified by their past history for similar events, and other factors, including the severity of airflow limitation, poor health status, age, presence of emphysema, and leukocytosis [13].
These observations are not without implications for the development of strategies aiming at the prevention of exacerbations, events that not only accelerate the progression of the disease but can also involve more organs and functions, thus increasing the risk of death. In fact, in the course of these events, a significant increase (death-related) of the plasma levels of the cardiac markers and an increase in the platelet aggregation, unrelated to smoke exposure, are observed [14-17]. Furthermore, a recent study [18] has demonstrated that in the course of an acute event an increased dispersion of the ECG P wave is noted, indicating a non-homogeneous atrial repolarisation with an increased risk of supraventricular arrhythmias: in the patients with frequent exacerbations, this anomaly is observable not only during the acute phase but also in conditions of stability. Therefore, the COPD exacerbations have serious consequences, not only of a pulmonary but also of a systemic nature, which justify the term of 'pulmonary attack' [19] and the 'frequent exacerbation' patients (or patients susceptible to exacerbations) may represent a type of patients with increased cardiovascular risk.

Biomarker analyses of the ECLIPSE cohort showed that baseline fibrinogen associates with exacerbation rate, besides exercise tolerance and mortality. Also IL-6 and CRP are significantly raised in COPD and are yet further raised during exacerbations; however, they display wide variability in stable subjects and therefore their value as useful biomarkers of COPD would appear to be limited [20].

\section{Lung function rapid and slow decliners: different phenotypes?}

Although historically COPD is considered a progressive disease, with an average decline of the respiratory function estimated to be of about $50 \mathrm{ml} /$ year compared with the physiological decline of about $30 \mathrm{ml} / \mathrm{year}$, the studies that have investigated longitudinally the individual variability of the functional loss have been quite limited. This aspect was

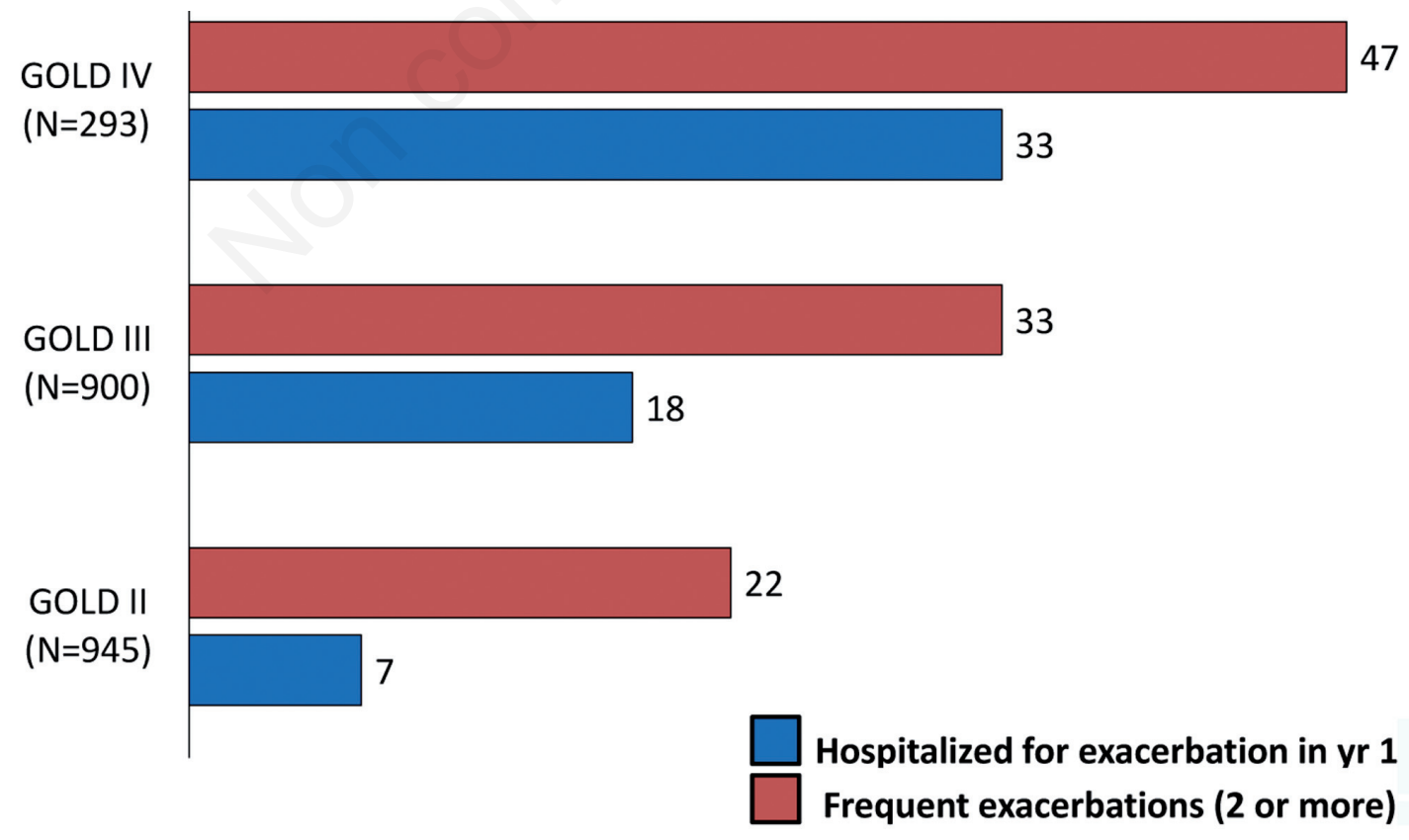

Figure 1. Association of disease severity with the frequency and severity of exacerbations during the first year of follow-up in patients with chronic obstructive pulmonary disease. 
explored in the ECLIPSE cohort [21], showing a variability in the functional decline in the course of the 3 year observation: in $38 \%$ of the cases it was above $40 \mathrm{ml} /$ year, in $31 \%$ from 21 to $40 \mathrm{ml} / y e a r$, in $23 \%$ the measurement of the FEV1 ranged from a $20 \mathrm{ml} /$ year decline to a 20 $\mathrm{ml} /$ year increase, in $8 \%$ of the patients the increase was above 20 $\mathrm{ml} /$ year (Figure 2).

Smoke turned out to be one of the main factors involved in the progression of the disease, as was the presence of emphysema, the frequency of exacerbations and, an unexpected and unexplained data, the bronchodilator reversibility. The decline was greater at the GOLD moderate stage (II) versus the very severe stage (IV), in line with the UPLIFT [22] and TORCH [23] studies. Although the role of pharmacological treatments of the progression of the disease is still debated, it cannot be denied that the decline is lower in patients under treatment.

It is interesting to observe how other studies have confirmed the heterogeneity of the progression of the disease [24,25]. For example, a longitudinal cohort study of 5 year duration [24] has reclassified the patients into 3 groups: with a fast decline, slow decline and stable. The only factor associated in an independent and statistically significant manner to a fast decline of the FEV1 proved to be the severity of emphysema, whereas other variables, such as the basic FEV1, the status of a smoker, and contrary to expectations, the exacerbation rate did not impact in any significant manner the functional loss. In this study, the stable patients, who represented about $25 \%$ of the cohort, showed a blood eosinophil count significantly higher than the 'decliner' patients and with a greater frequency of chronic bronchitis symptoms.

The data suggest that the clinical studies, where the functional decline is the primary outcome, should take into account the severity of the emphysema (predominant emphysematous COPD phenotype) as well as the fact that a high percentage of patients remain stable as regards spirometric parameters.

Lastly, none of the measured biomarkers has shown to be highly associated with functional decline, except for CC16, a molecular low weight protein produced by the Clara cells in the terminal bronchioles, with anti-inflammatory and immunosuppressive activity. However, the biological significance of this (weak) association is unknown [21].

\section{Is there a COPD phenotype with systemic inflammation?}

Persistent, low-level, systemic inflammation is thought to play a significant pathogenic role in many chronic diseases, including COPD [26-28]. Elevated circulating levels of several inflammatory markers, as C-reactive, protein (CRP), interleukins 6 (IL-6) and 8 (IL-8) have been reported in patients with COPD $[27,28]$. However, these studies were small, with large variability between patients and, most important, did not investigate the relationship between inflammation and relevant clinical outcomes of the disease.

The question whether the persistence of systemic inflammation in COPD could represent a distinct COPD phenotype was addressed by analysing the prevalence, temporal stability and network pattern of a panel of inflammatory biomarkers most often studied in COPD (WBC count, CRP, IL-6, IL-8, fibrinogen and TNFa) and their relationship with clinical characteristics and relevant outcomes at 3 years follow-up in the ECLIPSE cohort [29].

The results indicate that the patients with COPD show a distinct inflammatory pattern from that of the control subjects. In fact, despite the wide variability within the groups and the small absolute differences among the groups, a specific increase in some markers is observed especially fibrinogen, IL-6, PCR - compared with the control smokers and non-smokers (is it an inflammatory pattern specific of the COPD?), while the smokers without COPD show an increase in some specific biomarkers (IL-8, TNF-alpha) compared with both patients with COPD and non-smokers without COPD (Figure 3).

The possibility of a network among inflammatory mediators was also evaluated, where the link (network) between two markers was defined to be as existent if more than $1 \%$ of the subjects of the group shared an
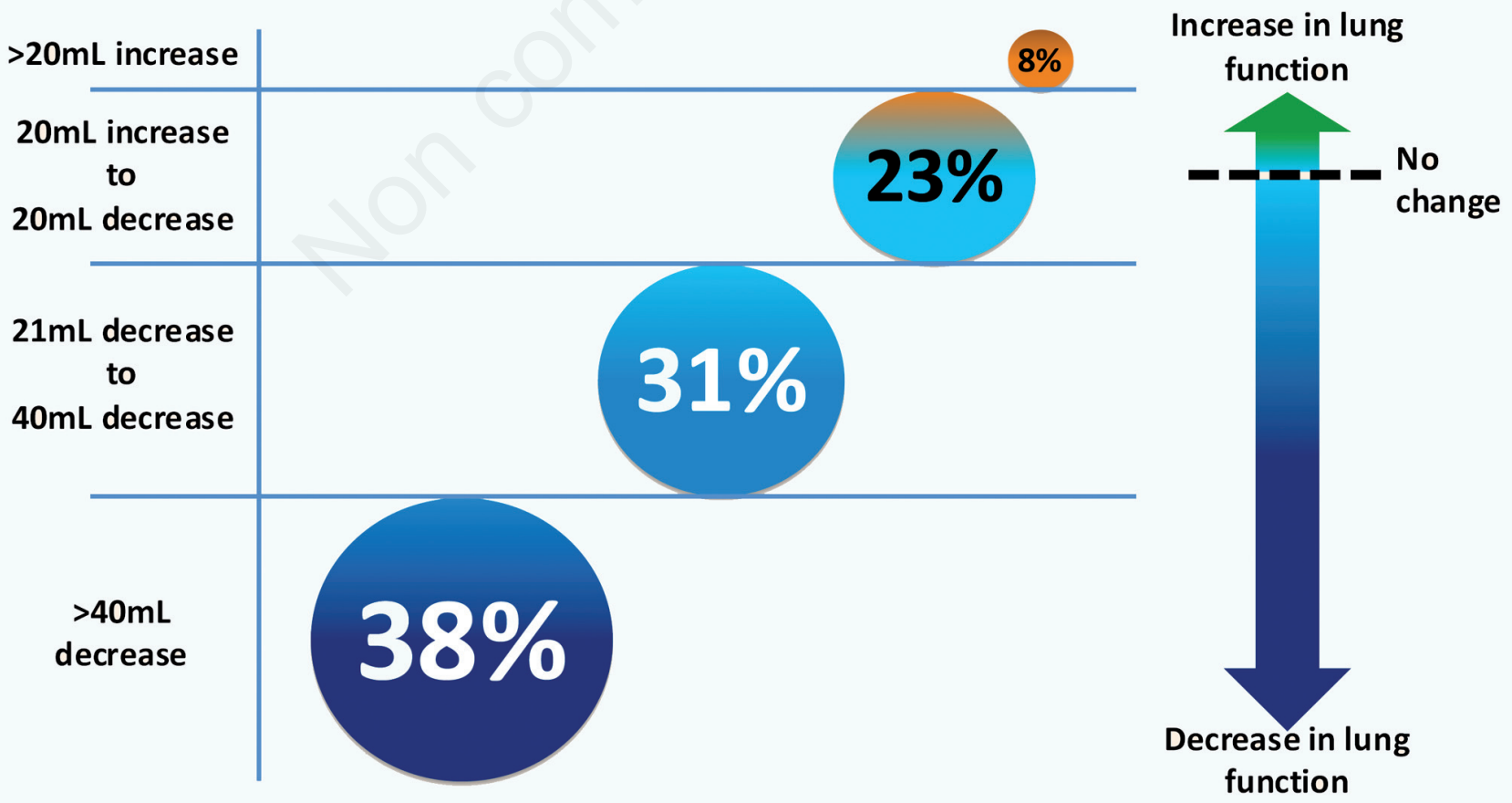

Figure 2. Distribution of estimated annual rates of change in forced expiratory volume in 1 second (FEV1) over a 3-year period in patients with chronic obstructive pulmonary disease. 


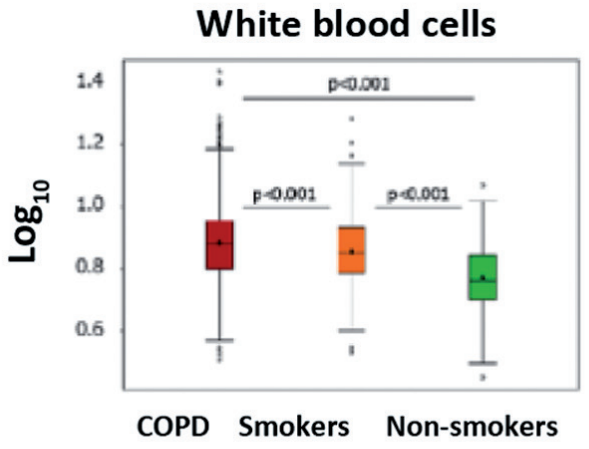

IL-8

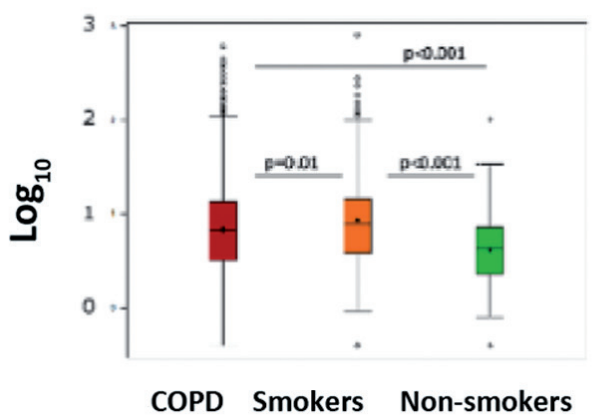

C-reactive protein

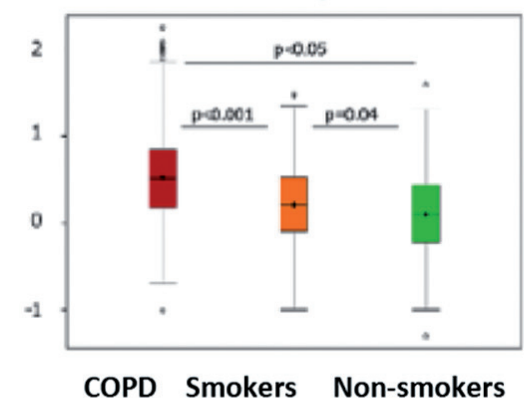

Fibrinogen

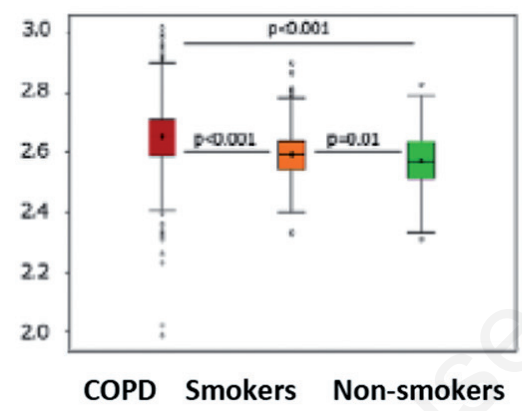

IL-6

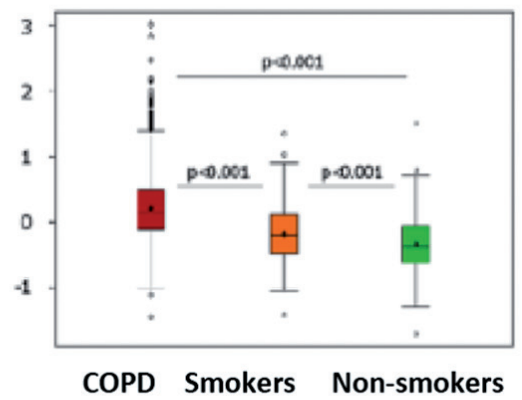

TNF $\alpha$

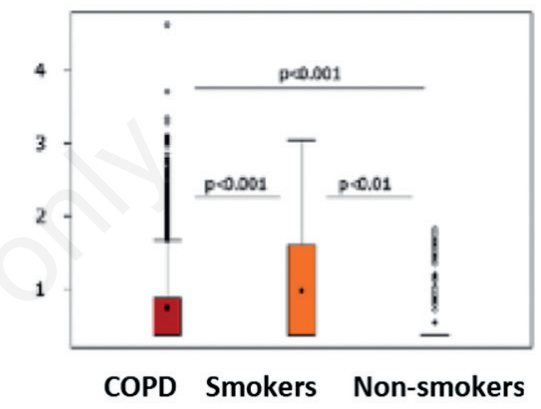

Figure 3. Box plot (log scale) of the different biomarkers determined at baseline in COPD patients, smokers with normal lung function and non-smokers.

above average increase in the haematic concentration of the two markers. The strength of the link was proportional to the number of subjects who showed a shared increase. As per Figure 4, a network of systemic inflammation (inflammome), intense and specific in patients with COPD compared with control groups, either smokers or nonsmokers, seems to emerge.

The longitudinal analysis, at 1 year, indicates that the subjects with consistently high levels of markers of systemic inflammation accounted for $16 \%$ of the sample. In this subgroup of patients with systemic 'permanent' flogosis, the risk for exacerbations is significantly higher compared with the 'less inflamed' patients, regardless of the level of bronchial obstruction. Interestingly, the factors that were most associated to the permanence of inflammation were found to be smoking and obesity.

A mortality analysis showed that the addition of a panel of selective systemic inflammatory markers measured at baseline significantly improved the ability of established clinical variables (BODE index) to predict mortality at three- and eight-year follow up [30,31].

Overall, the results suggest how the constant presence of systemic inflammation can identify a new, distinct phenotype of patient with COPD, a possible target for specific therapeutic strategies. The origin of the inflammation is unknown: it could derive from the airways (hypothesis of spill over of the mediators from the pulmonary tissue) or it could be of a systemic origin; the fact that the patients with persistent inflammation are more obese supports the latter hypothesis.

The role of systemic inflammation in COPD was further investigated by analyzing the prevalence and clinical characteristics associated with eosinophilic inflammation in the Eclipse cohort [32]. The results show that persistent eosinophillic inflammation (eosinophil counts $\geq 2 \%$ at all visits) appears to be a common feature of COPD, accounting for $37.4 \%$ of patients, and is associated with higher lung function values, less dyspnea and less progression of emphysema, suggesting that these subjects have less severe COPD. This requires confirmation in other cohorts. No impact on exacerbation rates was observed, at variance with other studies linking eosinophils with an increased risk of exacerbations [33,34].

\section{The reversibility to bronchodilator does not identify a COPD phenotype}

The analysis of reversibility to bronchodilator in the ECLIPSE cohort has shown, over the 3 year observation, that this parameter follows a rather fluctuating pattern in most patients [35]. In the patients with a constant pattern, no differences have emerged in terms of mortality, hospitalisations and exacerbations among those who were found at each assessment (at least 3), to be 'reversible' and 'non reversible'. Therefore, the reversibility to bronchodilator does not show the necessary requirements of stability and association to clinical relevant outcomes in order to configure a phenotype of patients with COPD.

\section{Asthma-COPD overlap (ACO)}

Asthma-COPD Overlap (ACO) has been recently defined as a new pathological entity [36]. There is evidence of an increasing prevalence of patients in whom the two diseases - asthma and COPD - coexist or in which one condition evolves into the other, leading to the pathological condition named ACO [36,37]. This occurs mainly in elderly patients suffering from asthma for a long time, especially if smokers. Recent estimations indicate that up to $24 \%$ of patients with COPD will additionally report a history of asthma, with an increasing trend in the elderly population (up to $50 \%$ in those aged over 70 years) $[38,39]$.

Patients with ACO have distinct clinical features (poorer health related quality of life, higher exacerbation rate, more rapid disease progression) 
Non-smokers

$(n=202)$
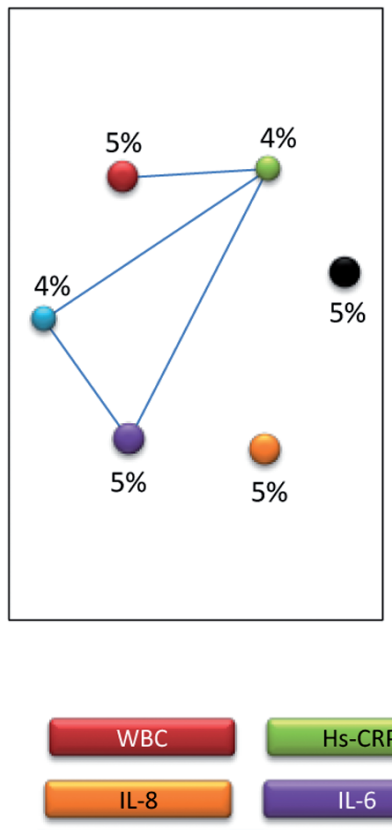

$1-5 \%$
Smokers

$(n=297)$

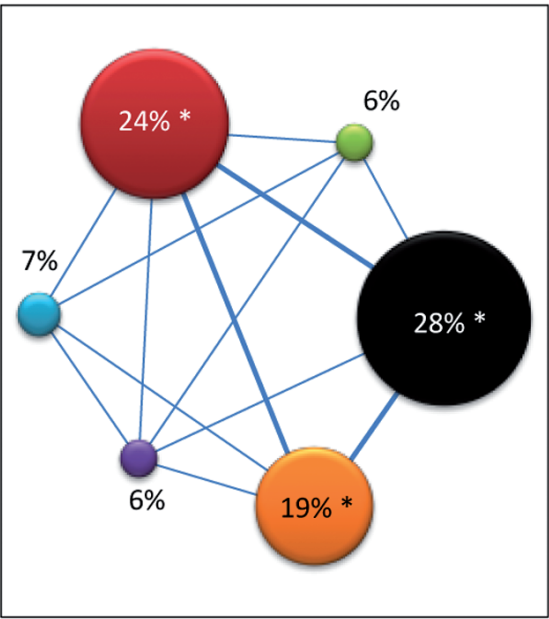

${ }^{*} \mathrm{p}<0.001$ vs Non-smokers
COPD

$(n=1755)$

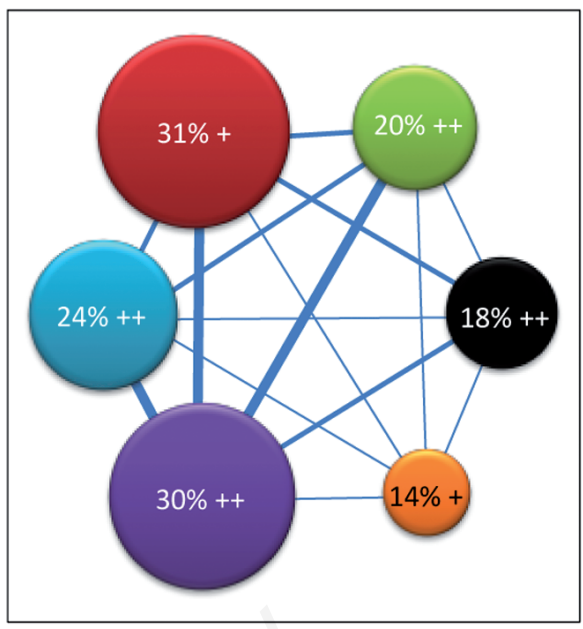

$+p<0.05 ;++p<0.001$ vs Smokers

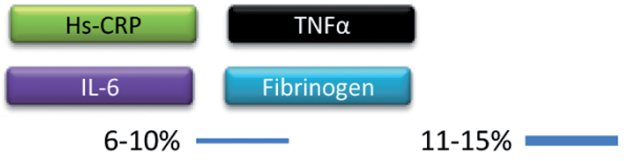

Figure 4. Network layout of the systemic inflammatory response (inflammome) in non-smokers $(\mathrm{n}=202)$, smokers with normal lung function $(\mathrm{n}=297)$ and COPD patients $(\mathrm{n}=1755)$ at recruitment. Each node of the network corresponds to one of the six inflammatory biomarkers determined in this study (see color code), and its size is proportional to the prevalence of abnormal values ( $95^{\text {th }}$ percentile of nonsmokers) of that particular biomarker in that particular group of subjects (precise figure shown inside of each node). Two nodes are linked if more than $1 \%$ of subjects in the network share abnormal values of these two biomarkers, its width being proportional to that proportion.

and possible genetic risk factors then subjects with asthma or COPD alone [36], suggesting that they may represent a distinct clinical phenotype. ECLIPSE offers an opportunity to examine ACO in a large well-characterized COPD cohort followed over 3 years [40]. For the primary definition, patients were considered to have ACO if they reported a history of asthma. Besides, other additional features of airways disease taken into consideration were bronchodilator reversibility at recruitment, atopy, wheeze, presence of current asthma. The results show that patients with ACO according to the primary definition were $25 \%$ of the cohort, were slightly younger, more often female, experience more exacerbations, had more frequently co-morbidities (osteoporosis, osteoarthritis, reflux/heartburn, depression, autoimmune disease) and used more respiratory medications and statins as compared with COPD patients without ACO [40]. These findings confirm prior research showing a worse health-status in patients with ACO than in patients with COPD [36]. In addition, the observation that plasma fibrinogen and serum TNF $\alpha$. concentration are slightly higher in ACO is consistent with its association with worse outcomes in COPD [29]. As regards the inflammatory pathway, there is limited evidence showing that Th2 allergic inflammation is active in ACO and may drive these clinical differences [41]. To explore this aspect, a multi-omics approach, including protein biomarkers, genome-wide association testing, and gene expression data, was used in subset of patients from two large cohort studies - COPDGene and ECLIPSE - to identify inflammatory patterns in ACO compared to COPD alone [42]. All subjects were current or former smokers with COPD (defined as $\mathrm{FEV}_{1} / \mathrm{FVC}<0.70$ and $\mathrm{FEV}_{1}<80 \%$ predicted). ACO was defined as subjects with COPD who additionally reported a history of asthma that was diagnosed by a physician before the age of 40 . Significantly greater values for biomarkers as SRAGE, CRP, and IgE levels were observed in ACO subjects compared to those with COPD alone, whereas peripheral eosinophils were similar in the two groups. ACO subjects demonstrated also significantly greater expression levels of several genes, including TLR10 ( $\mathrm{P}=0.0002)$, a gene involved in the innate immunity following viral infection that has previously been associated with asthma.

As regards the genome-wide association testing, the top SNP from the ECLIPSE population was near the gene FOXP1, that is involved in epithelial cell development in the lung as a negative regulator of follicular helper $\mathrm{T}$ cells. Overall, active inflammatory pathways present in ACO compared to COPD alone have been identified by a multi-omics approach. ACO subjects demonstrate increased IgE and innate immune gene expression.

These observations suggest that patients with the overlap phenotype may have inflammation which is more responsive to corticosteroids than patients with COPD alone and possibly a better prognosis. Randomized controlled clinical trials exclude smoker asthmatics and patients with overlapping asthma and COPD: therefore, the data on efficacy of treatment in this clinical population are lacking.

\section{New technologies and COPD phenotyping}

The metabolomic approach, combined with the proteomics and genomics, plays an increasingly important role in the biomedical research field. Metabolomics consists of the determination of the complete set of metabolites of a biological organism which are the final products of its gene expression. Therefore, this methodology may provide quick information on the response mechanisms of living systems to gene changes, to patho-physiological stimuli and to environmental influences, thus facilitating also the discovery and characterisation of the biomarkers. 
The metabolomic analysis of the ECLIPSE cohort, the widest to be assessed to date for this type of analysis, has shown that the metabolomic serum profile of the patients with COPD correlates with the different characteristics of spirometric severity, emphysema, systemic inflammation and BMI [30], thus providing additional information on patients phenotyping. For example, in patients with emphysema, an increase in creatinine and amino-acid precursor is observed reflecting a reduced mitochondrial function, in line with recent observations of a block of the electron transport chain with the production of free radicals also at the initial stages of the disease [43].

Studies on genetic polymorphisms have been carried out in cohorts of patients with COPD, including ECLIPSE, aiming at identifying the genetic determinants of emphysema: the results have led to the identification of a variant (SNP) of the BICD1 gene, associated with susceptibility to emphysema [44]. These variants are associated also with the length of the telomeres: this suggests that some mechanisms of accelerated cellular ageing are involved in the pathogenesis of emphysema. Other analyses have led to identifying genetic variants associated with different characteristics of the COPD [45-50]: i) limitation to the airflow (CHRNA3/5, IREB2, HHIP, FT0); ii) emphysema (CHRNA3/5, BICD1); iii) exacerbation frequency (HHIP); iv) BMI (e.g., HHIP, FTO); v) plasma levels of two proteins secreted from the lungs and related to COPD, CC16 and SP-D. Many of these associations were suggestive rather than genomewide significant and will require replication in additional studies.

Because of the amount of information that is starting to be produced, one of the biggest challenges, once the genomic data for particular phenotypes are available, is to go beyond the macro-molecular sequences in order to reach a higher level of integration, in a biological prospective such as clinical prognosis and response to medicinal products. The final objective of this integration is the possibility of identifying the subjects who are predisposed to COPD and to know which polymorphisms may affect their prognosis and their responsiveness to treatment.

\section{Conclusions}

The ECLIPSE study, carried out with a cohort characterised by subjects who were followed for 3 years, has provided a more clear vision of COPD as a complex and heterogeneous pathology, with variable progression, characterised by clinically important distinct types of patients, or phenotypes, who may have different prognosis or treatment requirements.

The complexity of the disease suggests that the assessment of its severity in patients requires a multidimensional approach. Within this context, the GOLD guidelines, in the 2011 edition, have proposed a new model for the assessment of the severity of the COPD and for its pharmacological management, which took into account, in addition to the FEV1 value, also the level of symptoms and the risk of exacerbations. This represents a major change since we have moved from a purely functional assessment to an assessment of clinical risk. Since the ECLIPSE study has shown that the main predictive factor of frequent exacerbations is a history of prior events, an accurate investigation of the clinical history related to exacerbations becomes highly important for the characterisation of patients and for the choice of treatment. So, given the clinical and economic importance of these events, frequent exacerbators should be targeted for effective exacerbation prevention, which is now recognized as a primary goal of maintenance therapy for COPD [1]. There is evidence from large clinical studies that the treatment with an inhaled corticosteroid-LABA combination significantly decreases the annual rate of exacerbations in comparison with the LABA alone [23,51,52]. A metaanalysis by Nannini et al. confirmed these findings [53]. Therefore, from available evidence, ICS/LABA combinations give the best results in patients with moderate or severe obstruction and risk of exacerbations. For the infrequent exacerbator phenotype, the treatment could based mainly on long-acting bronchodilators, alone or in combination.

As for the identification of biomarkers capable of not only identifying the subjects with an increased risk for the disease but also capable of identifying the phenotypes, predicting the severity or the progression of the disease and the responsiveness to the treatment, it emerges, from an analysis of the ECLIPSE study to date, that no single biomarker seems to fully meet all the necessary requirements. However, some potential markers of the progression of the disease have been identified, the validation of which requires the replication of the data in other cohorts of patients with COPD.

More studies are needed, particularly genetic studies, to reach a higher degree of integration of the genomic data with the possibility of identifying the subjects who are predisposed to COPD and to know which polymorphisms can affect phenotype, prognosis and responsiveness to treatment.

\section{References}

1. GOLD (Global Initiative for Chronic Obstructive Lung Disease). Global strategy for the diagnosis, management and prevention of COPD. 2014. Available from: www.goldcopd.com

2. Buist AS, McBurnie MA, Vollmer WM, et al. International variation in the prevalence of COPD (the BOLD Study): a population-based prevalence study. Lancet 2007;370:741-750.

3. Lopez AD, Murray CC. The global burden of disease, 1990-2020. Nat Med 1998;4:1241-1243.

4. Celli BR, Thomas NG, Anderson JA, et al. Effect of pharmacotherapy on rate of decline of lung function in chronic obstructive pulmonary disease results from the TORCH Study. Am J Respir Crit Care Med 2008;178:332-338.

5. Burrows B, Fletcher CM, Heard BE et al. The emphysematous and bronchial types of chronic airways obstruction. A clinicopathological study of patients in London and Chicago. Lancet 1966;1:830-5.

6. American Thoracic Society. Standards for the diagnosis and care of patients with chronic obstructive pulmonary disease. Am J Crit Care Med 1995;152:S77-121.

7. Pistolesi M, Carmiciottoli G, Paoletti M, et al., Identification of a predominant COPD phenotype in clinical practice. Nat Med 2008; 102:367-376.

8. Burgel PR, Paillasseur HL, Caillaud D, et al., Clinical COPD phenotypes: a novel approach using principal component and cluster analyses. Nat Med 2010;36:531-539.

9. Weatherhall M, Travers J, Shirtcliff PM, et al. Distinct clinical phenotypes of airways disease defined by cluster analysis. Nat Med 2009;34:812-818.

10. Garcia-Aymerich, Gomez FP, Benet M, et al., Identification and prospective validation of clinically relevant COPD subtypes. Thorax 2011;66:430-437.

11. Agusti A, Calverley PMA, Celli R, et al., Characterization of COPD heterogeneity in the ECLIPSE cohort. Nat Med 2010;11:122-136.

12. Hurst JR, Vestbo J, Anzueto A et al., Susceptibility to exacerbations in chronic obstructive pulmonary disease. N Engl J Med 2010; 363:1128-1138.

13. Müllerova H, Maselli DJ, Locantore N et al., Hospitalized exacerbations of COPD: risk factors and outcomes in the ECLIPSE cohort. Chest 2015;147:999-1007.

14. Maclay JD, McAllister DA, Johnston S, et al., Increased platelet activation in patients with stable and acute exacerbation of COPD. Thorax 2011;66:769-774. 
15. Høiseth AD, Neukamm A, Karlsson BD, et al. Elevated high-sensitivity cardiac troponin $\mathrm{T}$ is associated with increased mortality after acute exacerbation of chronic obstructive pulmonary disease. Thorax 2011;66:775-781.

16. McAllister DA, Maclay JD, Mills NL, et al. Diagnosis of myocardial infarction following hospitalisation for exacerbation of COPD. Eur Respir J 2012;39:1097-1103.

17. Chang CL, Robinson SC, Mills GD, et al. Biochemical markers of cardiac dysfunction predict mortality in acute exacerbations of COPD. Thorax 2011;66:764-768.

18. Fabbri LM, Beghé B, Agusti A. Cardiovascular mechanisms of death in severe COPD exacerbation: time to think and act beyond guidelines. Thorax 2011;6:745-747.

19. Bhatt SP, Nanda Sand Kintzer JS. Arrhythmias as trigger for acute exacerbations of chronic obstructive pulmonary disease. Respir Med 2012;106:1134-1138.

20. Dickens JA, Miller BA, Edwards LD, et al. COPD association and repeatability of blood biomarkers in the ECLIPSE cohort. Respir Res 2011;12:146-156.

21. Vestbo J, Edwards LD, Scanlon PD, et al. Changes in forced expiratory volume in 1 second over time in COPD. New Engl J Med 2011;365:1184-92.

22. Tashkin DP, Celli B, Senn S, et al. 4-year trial of tiotropium in chronic obstructive pulmonary disease New Engl J Med 2008;359:1543-1554.

23. Calverley PMA, Anderson JA, Bartolome C, et al. Salmeterol and fluticasone propionate and survival in Chronic Obstructive Pulmonary Disease. N Engl J Med. 2007;356:775-78

24. Nishimura M et al., Annual change in pulmonary function and clinical phenotype in chronic obstructive pulmonary disease. Am J Respir Crit Care Med 2012;185:44-52.

25. Casanova C, de Torres JP, Aguirre-Jaime A, et al. The progression of chronic obstructive pulmonary disease is heterogeneous: the experience of the BODE cohort. Am J Respir Crit Care Med 2011;184:1015-1021.

26. De Martinis M, Franceschi C, Monti D, Ginaldi L. Inflamm-ageing and lifelong antigenic load as major determinants of ageing rate and longevity. FEBS Lett 2005;579: 035-2039.

27. Fabbri LM, Rabe KF. From COPD to chronic systemic inflammatory syndrome? Lancet 2007;370:797-799.

28. Gan WQ, Man SF, Senthilselvan A, Sin DD. Association between chronic obstructive pulmonary disease and systemic inflammation: a systematic review and a meta-analysis. Thorax 2004;59:574-580.

29. Agusti A, Edwards LD, Rennard SI et al. Persistent systemic inflammation is associated with poor clinical outcomes in COPD: a novel phenotype. PloS One 2012;7:e37483.

30. Celli B, Locantore N, Yates L, et al. Inflammatory biomarkers improve clinical prediction of mortality in chronic obstructive pulmonary disease. Am J Respir Crit Care Med 2012;185:1065-1072.

31. Locantore N, Celli B, Agusti A, et al. The BODE index and BODE plus biomarkers as predictors of long term survival in COPD. Am J Respir Crit Care Med 2016; 193: A3482.

32. Singh D, Kolsum U, Brightling BE et al., Blood eosinophil measurements may be useful for selecting COPD patients for different therapeutic approaches. Eur Respir J 2014; 44: 1697-700.

33. Pascoe S, Locantore N, Dransfield MT, et al. Blood eosinophil counts, exacerbations, and response to the addition of inhaled fluticasone furoate to vilanterol in patients with chronic obstructive pulmonary disease: a secondary analysis of data from two parallel randomised controlled trials. Lancet Respir Med 2015;3:435-442.

34. Siddiqui SH, Guasconi A, Vestbo J, et al. Blood eosinophils: a biomarker of response to extrafine beclomethasone/formoterol in chronic obstructive pulmonary disease. Am J Respir Crit Care Med 2015;192:523-525.
35. Albert P, Agusti A, Edwards L, et al. Bronchodilator responsiveness as a phenotypic characteristic of established chronic obstructive pulmonary disease. Thorax 2012;67:701-708.

36. Global Initiative for Asthma (GINA). 2015 Asthma, COPD and Asthma-COPD Overlap Syndrome (ACOS). Available from: http://ginasthma.org

37. De Marco R, Pesce G, Marcon A, et al. The coexistence of asthma and chronic obstructive pulmonary disease (COPD): prevalence and risk factors in young, middle-aged and elderly people from the general population. PLoS One 2013;8:e62985.

38. Hardin M, Cho M, McDonald ML, et al. The clinical and genetic features of COPD-asthma overlap syndrome. Eur Respir J 2014; 44:341-350.

39. Soriano JB, Davis KJ, Coleman B, et al. The proportional Venn diagram of obstructive lung disease: two approximations from the United States and the United Kingdom. Chest 2003;124:474-481.

40. Donohue JF, Herje N, Crater G, Rickard K. Characterization of airway inflammation in patients with COPD using fractional exhaled nitric oxide levels: a pilot study. Int J Chron Obstruct Pulmon Dis 2014;9:745-751.

41. Wurst KE, Rheault TR, Edwards L et al., A comparison of COPD patients with and without ACOS in the ECLIPSE study. Eur Respir J 2016; 47: 1559-1562.

42. Hardin M, Cho M, McDonald ML, et al. Asthma-COPD overlap subjects demonstrate distinct patterns of inflammation compared to those with COPD alone. Am J Respir Crit Care Med 2016; 193: A7473.

43. Puente-Maestu L, Pérez-Parra J, Godoy R, et al. Abnormal mitochondrial function in locomotor and respiratory muscles of COPD patients. Eur Respir J 2009;33:1045-1052.

44. Kong X, Cho MH, Anderson W et al., Genome-wide association study identifies BICD1 as a susceptibility gene for emphysema. Am J Respir Crit Care Med 2011;183:43-49.

45. Pillai SG, Kong X, Edwards LD, et al. Loci identified by genomewide association studies influence different disease-related phenotypes in chronic obstructive pulmonary disease. Am J Respir Crit Care Med 2010;182:1498-1505.

46. Faner R, Rojas M, MacNee W, et al. Abnormal lung aging in chronic obstructive pulmonary disease and idiopathic pulmonary fibrosis. Am J Respir Crit Care Med 2012; 86:306-313.

47. Schols AM, Broekhuizen R, Weling-Scheepers CA, et al. Body composition and mortality in chronic obstructive pulmonary disease. Am J Clin Nutr 2005;82:53-59.

48. Wan ES, Cho MH, Boutaoui N, et al. Genome-wide association analysis of body mass in chronic obstructive pulmonary disease. Am J Respir Cell Mol Biol 2011;45:304-310.

49. Kim DK, Cho MH, Hersh CP, et al. Genome-wide association analysis of blood biomarkers in chronic obstructive pulmonary disease. Am J Respir Crit Care Med 2012;186:1238-1247.

50. Faner R, Tal-Singer R, Riley JH, et al. Lessons from ECLIPSE: a review of COPD biomarkers. Thorax 2014;69:666-672.

51. Wencker M, Glaab T, Vogelmeier C. Impact of salmeterol/fluticasone propionate versus salmeterol on exacerbations in severe chronic obstructive pulmonary disease. Am J Respir Crit Care Med 2007;175:144-149.

52. Dransfield MT, Bourbeau J, Jones PW et al. Once-daily inhaled fl uticasone furoate and vilanterol versus vilanterol only for prevention of exacerbations of COPD: two replicate double-blind, parallel-group, randomized controlled trials. Lancet Respir Med 2013;1:210-223.

53. Nannini L, Lasserson T, Poole P. Combined corticosteroid and longacting beta agonist in one inhaler versus long-acting beta-agonists for chronic obstructive pulmonary disease. Cochrane Database Syst Rev 2012;9:CD006829. 